\title{
APPLICATION OF A PERIODIC FOURIER MODAL METHOD TO METAL-PLATE LENS ANTENNAS
}

\author{
K. Edee*, I. Fenniche*, G. Granet*, J. P. Plumey*, E. Jehamy ${ }^{\dagger}$, M.Ney $^{\dagger}$ \\ *Lasmea, UMR 6602, 24 Av. des Landais 63177 Aubière cedex France. \\ ${ }^{\dagger}$ LEST, CS 83818, 29238 Brest Cedex3, France. \\ Corresponding author: Gerard.GRANET@lasmea.univ-bpclermont.fr
}

Keywords: Metal plate lens, Fourier Modal Method.

\begin{abstract}
This paper is devoted to a modal method for fast design of electrically large metal-plate lens structures. The method is based on Fourier Modal Method (FMM). The lens consists of stacked parallel-plate waveguides. The lens is considered as a medium with discontinuities described by Dirac Delta functions.
\end{abstract}

\section{Introduction}

Collision avoidance radar systems will become standard equipment on vehicles in the near future. They operate at $76 \mathrm{GHz}$ and will be able to achieve short and longrange detection. Radar antenna specifications require high gain, low side-lobe level and vertical polarization. It has been shown that such requirements could be achieved with metal plate lens, also referred as artificial lens [1]. It consists of stacked parallel-plate waveguides. The distance between plates is chosen to minimize ohmic losses and insure $T E_{1}$ mode operation. Since the equivalent local effective refractive index of the waveguides is less than one, each plays the role to increase the phase velocity of the wave propagating through it. Figure 1 shows a two-dimensional model of an artificial lens. In normal beam operation for instance, high gain and low side lobe level must be achieved simultaneously. Thus, electromagnetic analysis and optimization techniques must be applied. Typically, input and output lens profiles can be determined for optimal performances. They are expressed in polynomial form and coefficients are the parameters to be found.

Generally, there are many unknowns, and Genetic Algorithms may provide an efficient tool to perform optimization. However since they require a great number of population evaluations, they are time consuming when they are combined with rigorous full-wave analysis. For the present structure, which is several wavelengths large, asymptotic methods like Physical Optics and Geometrical Optics (GO) are reliable. Indeed, it has been shown that simulated results generally compare favourably with experiments. However significant discrepancies for side lobe levels are observed. They are explained by the fact that GO based models do not account for diffraction by edges. In order to have a rigorous and fast algorithm, we have developed an in-house code based on the Aperiodic Fourier Modal Method (AFMM) widely used in the optical domain. This paper will present the method and show simulation results.

\section{FMM applied to perfect conducting parallel-plate medium with negligible thickness}

\subsection{Infinite parallel-plates medium: periodic scheme}

In Cartesian coordinates systems $(O x, y, z)$, we assume 2D feature $\left(\partial_{y}=0\right)$, as focusing only occurs in the $\mathrm{xOz}$-plane and TE-polarization. Hence the only non-zero components of the electromagnetic field are $E_{y}, H_{x}, H_{z}$. In this case of polarization, $E_{y}$ satisfies:

$$
\left[\partial_{x}^{2}+k_{0}^{2} \varepsilon(x)\right] E_{y}(x, z)=-\partial_{z}^{2} E_{y}(x, z)
$$

and the other components of the field may be expressed in terms of $E_{y}$ :

$$
\begin{aligned}
& \partial_{z} E_{y}(x, z)=-i \omega \mu_{0} H_{x}(x, z) \\
& \partial_{x} E_{y}(x, z)=i \omega \mu_{0} H_{z}(x, z)
\end{aligned}
$$

where $i^{2}=-1, \partial_{z}=\frac{\partial}{\partial_{z}}, \partial_{x}=\frac{\partial}{\partial_{x}}, k_{0}=\frac{2 \pi}{\lambda}, \omega$ is the angular frequency and $\mu_{0}$ is the permeability of vacuum. The term $\varepsilon(x)$ denotes the permittivity function of the medium.

Let us consider the case of perfect conducting parallelplate periodic medium (Fig. 2.1). Also, let assume $x_{q}=q d$ be the $x$-position of any plate. This parallelplate medium is replaced by a medium that ensures the same boundaries condition at $x_{q}$. The relative permittivity function of this equivalent medium is defined as follow:

$$
\varepsilon_{r}(x)=1-i \kappa \sum_{q} \delta\left(x-x_{q}\right)
$$

where $\delta$ is the Dirac distribution, $\kappa$ is a parameter which has to be chosen large enough to take into account the conductivity of the plates. Since the left side of Eq. 1 does not depend on $z$, we can seek solutions under the 


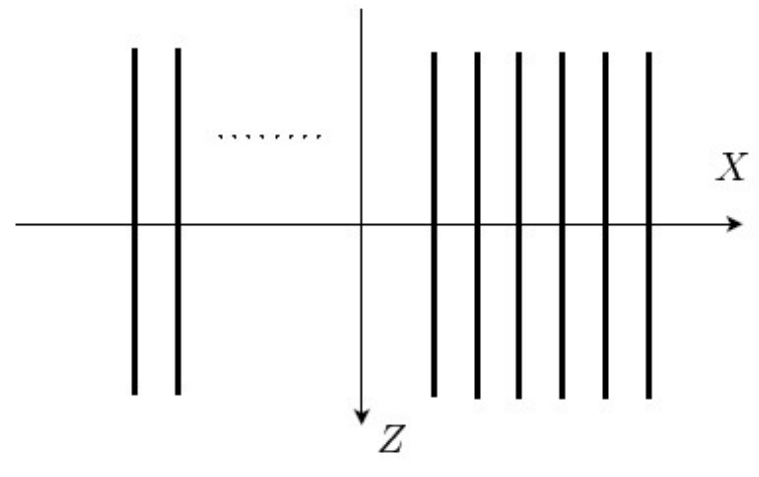

Figure 1: Infinite parallel-plates medium.

form $\phi(x, z)=\varphi(x) \exp \left(-i k_{0} \gamma z\right)$. Hence, Eq. 1 becomes an eigenvalue equation:

$$
£(x) \varphi(x)=k_{0}^{2} \gamma^{2} \varphi(x),
$$

where $£(x)=\partial_{x}^{2}+k_{0}^{2} \varepsilon(x)$. A periodic boundaries condition in the $x$-direction is used by introducing a Fourier basis expansion. The functions $\varphi(x), \varepsilon_{r}(x)$, and $1 / \varepsilon_{r}(x)$ are expanded into a sequence of Fourier basis functions $e_{n}(x)=\exp \left(-i k_{0} \alpha_{n} x\right)\left(\alpha_{n}=\frac{n \lambda}{d}\right)$. Galerkin's moment method is used to solve Eq. 3 .

\subsection{Finite parallel-plates medium: non-periodic scheme}

In the case of a finite number of parallel-plates, it is necessary to introduce free-space radiation conditions in $x$ direction. The matter is to find numerically the solutions of Eq. 3, when the relative permittivity function is defined as follow:

$$
\varepsilon_{r}(x)=1-i \kappa \sum_{q=1}^{N} \delta\left(x-x_{q}\right), N \in \mathbb{N} .
$$

This is done by introducing PMLs (Perfect Match Layers) through the concept of complex coordinate [2] and within the framework of FMM. The $x$-coordinate inside the PML is mapped to a complex variable $\widetilde{x} \mathrm{x}$-domain as $x \mapsto \widetilde{x}=$ $\int_{0}^{x} s(x) d x$, where:

$$
s(x)= \begin{cases}(1-i \eta) x & \text { if } x \in\left[\begin{array}{ll}
0 & e_{p m l}
\end{array}\right], \eta>0 \\
x & \text { otherwise. }\end{cases}
$$

With this change of variable, $\partial_{\widetilde{x}}=\frac{\partial x}{\partial \widetilde{x}} \frac{\partial}{\partial x}=\frac{1}{s(x)} \partial_{x}$, Eq. 3 becomes $£(x) \varphi(\widetilde{x}(x))=\gamma^{2} \varphi(\widetilde{x}(x))$, where :

$$
£(x)=\frac{1}{k_{0}^{2}}\left[\frac{1}{s(x)} \partial_{x} \frac{1}{s(x)} \partial_{x}+k_{0}^{2} \varepsilon(\widetilde{x}(x))\right]
$$

Even though the eigenvectors of $£(x)$ are expanded in Fourier basis functions:

$$
\varphi(x)=\lim _{N \mapsto+\infty} \sum_{n=-N}^{N} \Phi_{n} e_{n}(x),
$$

the free-space conditions are taken into account efficiently in the domain inside the PML. Since the $£(x)$ eigenvalues are computed through their square values $\gamma^{2}$, we can find at least two types of eigenvectors $\varphi^{+}(x)$ and $\varphi^{-}(x)$ such as $\phi(x, z)=\varphi^{+}(x) \exp \left(-i k_{0} \gamma^{+} z\right)$ and $\left(\gamma^{+}\right)^{2}=\left(\gamma^{-}\right)^{2}=\gamma$. $\gamma^{+}$and $\gamma^{-}$are such that $\phi(x, z)$ satisfy an outgoing wave condition when $z \mapsto_{-}^{+} \infty$, i.e. imaginary part of $\gamma^{+}$is negative while the imaginary part of $\gamma^{-}$is positive.

\section{Application to a metal-plates lens an- tennas}

The analysis of the metal-plate lens antennas (Fig. 2) is quite analogous to that used in the finite parallel-plate medium. The metal-plate lens antennas can be viewed as

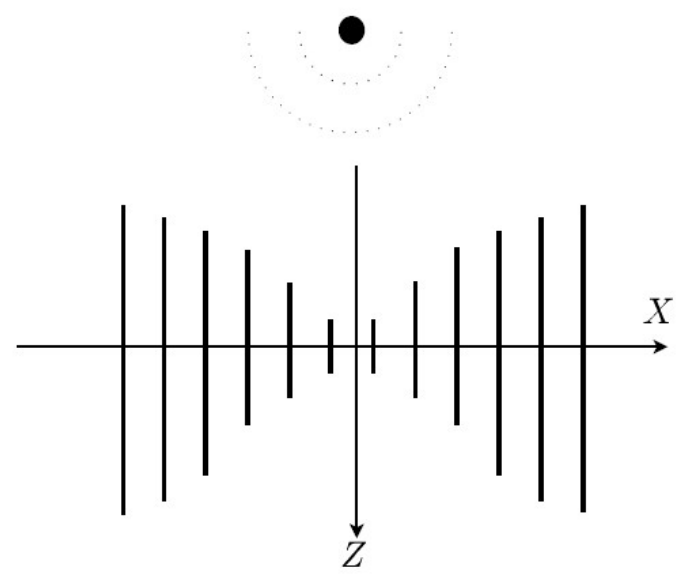

Figure 2: Two-dimensional artificial lens.

a stack of layers of finite parallel-plates medium (Fig. 3) and the propagation of the electromagnetic wave through the lens can be analyzed as a coupled mode-process. The

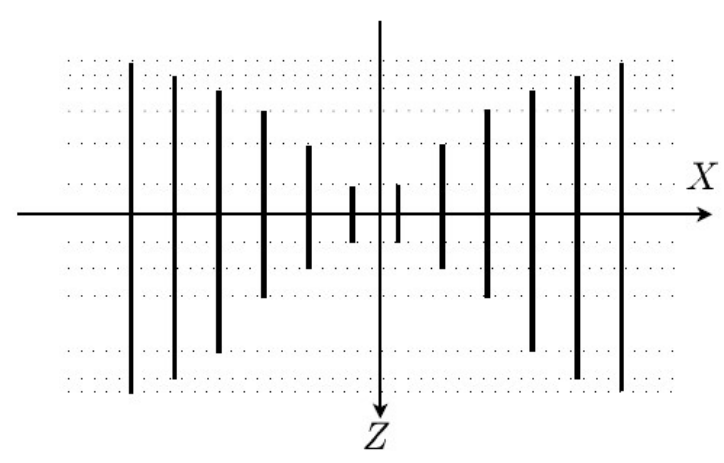

Figure 3: Metal-plate lens viewed as a stack of layers of finite parallel-plates medium.

lens is divided into layers denoted by $C^{j}, j \in \mathbb{N}$. Each layer $C^{j}$ is characterized by its thickness $h^{j}$ and its relative 
permittivity function $\varepsilon_{r}^{j}$ :

$$
\varepsilon_{r}^{j}(x)=1-i \kappa \sum_{q=1}^{N^{j}} \delta\left(x-x_{q}^{j}\right), N^{j} \in N
$$

In each $C^{j}$ layer, $E_{y}^{j}(x)$ is represented as a superposition of $£^{j}(x)$ eigenvectors of the form:

$$
E_{y}^{j}(x, z)=\sum_{m=1}^{\infty} R_{m}^{+j} \varphi_{m}^{{ }_{m}^{+j}}(x) \exp \left(-i k_{0} \gamma_{m}^{+j} z\right)
$$

where, $R_{m}^{+j}$ are the spectral amplitude of the field. The eigenvalues roots, $\gamma_{m}^{+j}$ and $\gamma_{m}^{-j}$ are such that the field satisfy the Sommerfeld's outgoing conditions for $z \mapsto_{-}^{+} \infty$ according to the $\exp \left(-i k_{0} \gamma_{m}^{j} z\right)$ dependence. Naturally the associated eigenvectors are $\varphi_{m}^{-j}$. Inserting Eq. 7 in Eq. 9, we establish that:

$$
E_{y}^{j}(x, z)=\sum_{m=1}^{\infty} R_{m}^{+j} \exp \left(-i k_{0} \gamma_{m}^{+j} z\right) \sum_{m=-\infty}^{+\infty} \Phi_{n m}^{+j} e_{n}(x),
$$

thus:

$H_{x}^{j}(x, z)=\frac{1}{Z_{0}} \sum_{m=1}^{\infty} R_{m}^{-j} \exp \left(-i k_{0}{\gamma_{m}^{+j}}^{+j}\right) \sum_{n=-\infty}^{+\infty} \gamma_{m}^{+j} \Phi_{n m}^{+{ }_{n}^{j}} e_{n}(x)$

$H_{z}^{j}(x, z)=-\frac{1}{Z_{0}} \sum_{m=1}^{\infty} R_{m}^{+j} \exp \left(-i k_{0} \gamma_{m}^{+j} z\right) \sum_{n=-\infty}^{+\infty} \alpha_{n}^{j} \Phi_{n m}^{+j} e_{n}(x)$.

To be complete, we should mention briefly that the coupled mode-process is performed through S-matrix formulation by expressing the conservation of tangential components $E_{y}^{j}$ and $H_{x}^{j}$ at the interface of layers $C^{j}$ and $C^{j+1}$.

\section{Source line simulation [3]}

The 2D Green's function $G(x, z)$, periodic with respect to $x$, obeys the following equation:

$\partial_{z}^{2} G(x, z)+\partial_{x}^{2} G(x, z)+k_{0}^{2} \varepsilon_{0} G(x, z)=\delta\left(z-z_{0}\right) \delta\left(x-x_{0}\right)$.

By Fourier transform Eq. 13 becomes:

$$
\partial_{z}^{2} \widetilde{G}(\alpha, z)+k_{0}^{2} \varepsilon_{0}\left(1-\alpha^{2}\right) \widetilde{G}(\alpha, z)=e^{i k_{0} \alpha x_{0}} \delta\left(z-z_{0}\right)
$$

in the sense of distributions,

$$
\begin{array}{r}
\partial_{z}^{2} \widetilde{G}+k_{0}^{2} \varepsilon_{0}\left(1-\alpha^{2}\right) \widetilde{G}=\left[\partial_{z} G\right]_{z_{0}} \delta_{z_{0}}+[G]_{z_{0}} \delta_{z_{0}}^{\prime} \\
+\left\{\partial_{z}^{2} \widetilde{G}+k_{0}^{2} \varepsilon_{0}\left(1-\alpha^{2}\right) \widetilde{G}\right\}
\end{array}
$$

hence

$$
\left\{\partial_{z}^{2} \widetilde{G}+k_{0}^{2} \varepsilon_{0}\left(1-\alpha^{2}\right) \widetilde{G}\right\}=0,
$$

and

$$
\left\{\begin{array}{l}
{[G]_{z_{0}}=0} \\
{\left[\partial_{z} G\right]_{z_{0}}=e^{i k_{0} \alpha x_{0}}}
\end{array}\right.
$$

From Eq.16, $G(x, z)$ may be described in terms of eigenvector of $£(x)$ :

$$
G(x, z)=\sum_{m, n}^{\infty} A_{m}^{+} \exp \left(-i k_{0} \gamma_{m}^{+} z\right) \Phi_{n m}^{+} e_{n}(x),
$$

and Eq. (17) provides the numerical values of the spectral amplitude $A_{m}^{-}$.

\section{$5 \quad$ Numerical results}

We provide numerical examples to illustrate the effectiveness of our formulation. The lens is illuminated by a line source described in the previous section (see Fig. 2). Figure 4 represents an equiphase surface.

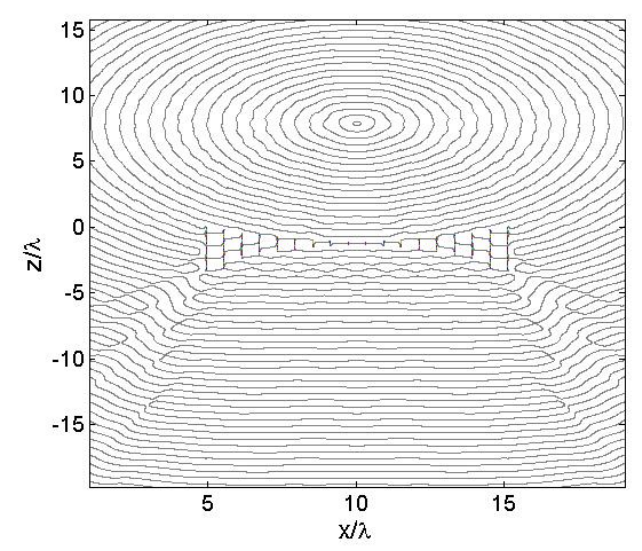

(a) line source located at the main focusing point.

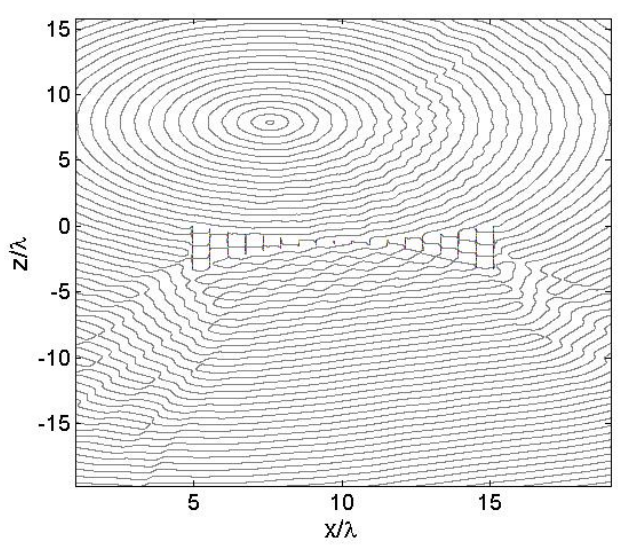

(b) line source located at a secondary focusing point.

Figure 4: metal plate lens illuminated by a line source: representation of the field phase.

Contributions from lens edges to the radiation pattern can be clearly observed. One can notice that the transmitted 


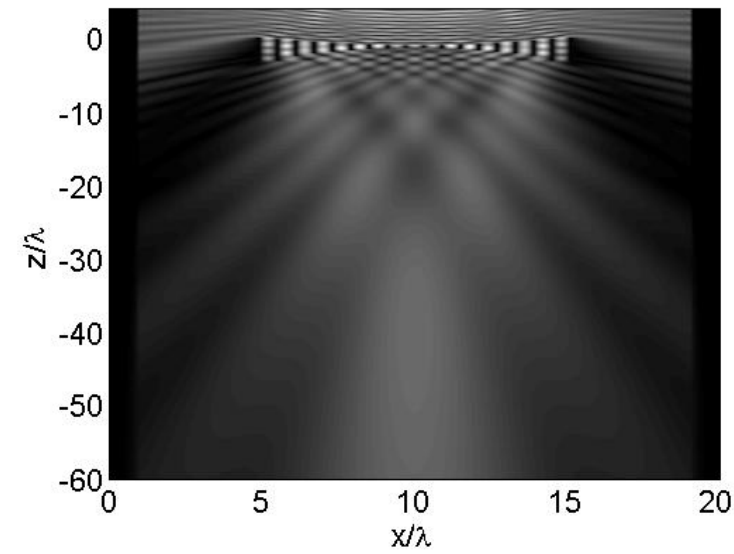

(a) line source located at the main focusing point.

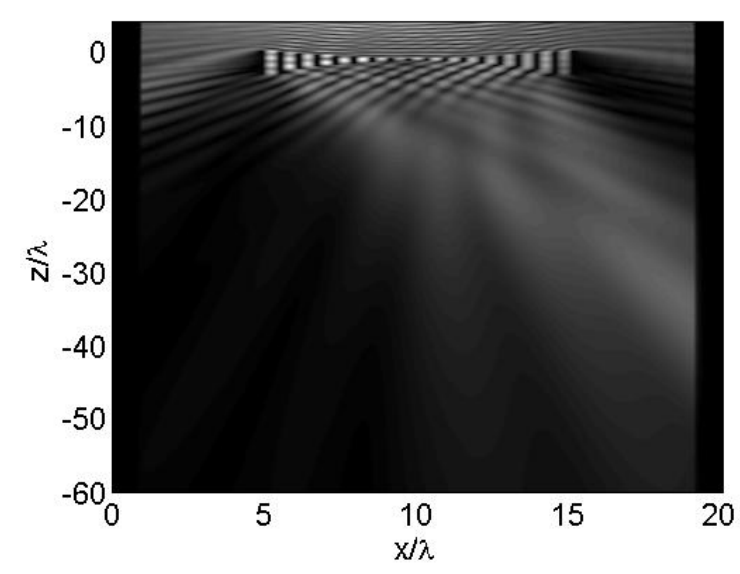

(b) line source located at a secondary focusing point.

Figure 5: metal plate lens illuminated by a line source: representation of the field amplitude.

wave is locally plane when the source is located at the lens main focusing point (Fig. 4(a)). In this case, the source is located on the lens symmetry axis. Hence, the wavefront is orthogonal to the system. Figure 5 shows the electric field magnitude. Figures 4(b) and 5(b) show the effects due to the source displacement towards the secondary focusing point. One can observe that the wavefront is tilted from the lens axis.

\section{Conclusion}

We have presented in this paper a rigourous simulation of the behaviour of a metallic artificial lens in the presence of a line source. The model is based on the Fourier modal method (FMM). The FMM introduces a periodical boundaries condition. However, the lens has a finished extend. To take into account the non-periodicity of the structure, PMLs were introduced by a modified complex coordinate. An application to an optimized lens shows that edges effects, du to the diffraction phenomena should be taken into account.

\section{References}

[1] F. Gallée , G. Landrac, M. Ney, "Artificial lens for third generation Automotive Radar Antenna at Millimeter-wave Frequencies", IEE Procedings, Microwaves-Antennas and Prop. Vol 150, Issue 6, pp 470-476, Dec 2003.

[2] W. C. Chew and W. H. Weedon, "A 3D perfectly matched medium from modified Maxwell's equations with stretched coordinates", Microwave Opt. Technol. Lett. 7, 599-604 1994.

[3] J. P. Plumey, K. Edee, G. Granet, "Modal expansion for the 2D Green's function in a non-orthogonal coordinates system", Progress In Electromagnetics Research 59, 101-112, 2006. 